\title{
Promoção da saúde e qualidade de vida: uma perspectiva histórica ao longo dos últimos 40 anos (1980-2020)
}

\author{
Health promotion and quality of life: a historical perspective of the \\ last two 40 years (1980-2020)
}

Paulo Marchiori Buss (http://orcid.org/0000-0002-9944-9195) ${ }^{1}$

Zulmira Maria de Araújo Hartz (http://orcid.org/0000-0001-9780-9428) ${ }^{2}$

Luiz Felipe Pinto (http://orcid.org/0000-0002-9888-606X) ${ }^{3}$

Cristianne Maria Famer Rocha (http://orcid.org/0000-0003-3281-2911) ${ }^{4}$

${ }^{1}$ Centro de Relações Internacionais em Saúde, Fiocruz. Av. Brasil 4365, Manguinhos. 21041-900 Rio de Janeiro RJ Brasil. paulo.buss@fiocruz.br

${ }^{2}$ Instituto de Higiene e Medicina Tropical, Universidade Nova de Lisboa. Lisboa Portugal. ${ }^{3}$ Programa de PósGraduação em Atenção Primária à Saúde, Faculdade de Medicina, Universidade Federal do Rio de Janeiro. Rio de Janeiro RJ Brasil. ${ }^{4}$ Departamento de Saúde Coletiva, Escola de Enfermagem, Universidade Federal do Rio Grande do Sul. Porto Alegre RS Brasil.

\begin{abstract}
This article updates the previous text of the main author published in 2000, revisiting the scientific evidence that reaffirms the contribution of health to the quality of life of individuals and populations. More than the access to health services of any quality, it is necessary to face determinants of health in its entirety, which requires healthy public policies, an effective intersectoral articulation of public power and mobilization of the population. The authors revisit the emergence and development of health promotion, focusing on the analysis of the most promising health strategies for the increase in quality of life, especially in societies with high social and health inequalities, as in the case of Brazil, reinforced by the recent pandemic of COVID-19. Such strategies were concretized on healthy municipalities and intersectoral actions, in health and in all policies which confront social determinants, through their own foundations and practices that are closely related to innovations in public management for integrated and sustainable local development, in view of the 2030 Agenda and its Sustainable Development Objectives (SDG).
\end{abstract}

Key words Health promotion, Quality of life, SDG, 2030 Agenda
Resumo Este artigo atualiza o texto anterior do autor principal publicado em 2000, revisitando as evidências científicas que reafirmam a contribuição da saúde para a qualidade de vida de indivíduos e populações. Mais do que o acesso a serviços de saúde de qualidade, é necessário enfrentar os determinantes da saúde em toda a sua amplitude, o que requer políticas públicas saudáveis, uma efetiva articulação intersetorial do poder público e a mobilização da população. Os autores revisitam a emergência e o desenvolvimento da promoção da saúde, centrando sua análise nas estratégias mais promissoras para o incremento da qualidade de vida propostas pelo setor saúde, sobretudo em formações sociais com alta desigualdade sociosanitária, como é o caso do Brasil, reforçada pela recente pandemia de COVID-19. É no movimento dos municípios saudáveis e em ações intersetoriais, na saúde em todas as políticas e no enfrentamentos dos determinantes sociais da saúde que tais estratégias se concretizam, através de seus próprios fundamentos e práticas, que estão estreitamente relacionados com as inovações na gestão pública para o desenvolvimento local integrado e sustentável, "vis a vis" a nova Agenda 2030 e seus Objetivos do Desenvolvimento Sustentável (ODS).

Palavras-chave Promoção da saúde, Qualidade de vida, ODS, Agenda 2030 


\section{Introdução}

As condições de vida e saúde melhoraram de forma contínua e sustentada na maioria dos países, graças aos progressos políticos, econômicos, sociais, ambientais e aos avanços na saúde pública e na medicina. Estudos de diferentes autores e relatórios sobre a saúde mundial ${ }^{1,2}$ e da região das Américas são conclusivos a respeito. $\mathrm{Na}$ América Latina, por exemplo, a expectativa de vida, nos últimos 20 anos, passou de 72,3 para 76,9 anos. Entretanto, as mesmas organizações são taxativas em apontar a permanência de profundas desigualdades entre os países, dentro deles, nas regiões e entre grupos sociais ${ }^{3}$.

Ao examinar as condições de morbimortalidade prevalentes nas Américas, verifica-se, a permanência de problemas já resolvidos em muitos lugares (determinadas doenças infecto-parasitárias e condições ligadas à infraestrutura urbana); o crescimento das doenças crônicas como o câncer, as enfermidades neurodegenerativas, cardio e cerebrovasculares e causas externas. Também surgem novos problemas (como a contemporânea COVID-19) aumento do uso de drogas, violência e agravos à saúde mental. A principal resposta social a tais questões tem sido investimentos crescentes em assistência médica curativa e individual, ainda que se identifique que medidas preventivas, promoção da saúde e melhoria das condições de vida sejam de fato as razões fundamentais para os avanços mencionados.

Este artigo pretende discutir a contribuição da promoção da saúde, como campo de conhecimento e de prática, para a qualidade de vida. Para tanto, discutem-se esses conceitos e algumas estratégias capazes de operacionalizar sua interação: políticas públicas saudáveis que exigem ação intersetorial e uma nova institucionalidade social que vem se materializando com as propostas de saúde em todas as políticas, municípios saudáveis, Agenda 2030 e seus Objetivos de Desenvolvimento Sustentável (ODS).

Este texto é uma versão atualizada do artigo de Paulo Marchiori Buss, de 2000: Promoção da saúde e qualidade de vida ${ }^{4}$.

\section{Saúde e qualidade de vida}

A influência da saúde sobre as condições e a qualidade de vida, e vice-versa, tem ocupado políticos e pensadores ao longo da história. No século XVIII, quando ocupava as funções de diretor geral de saúde pública da Lombardia austríaca, Johann Peter Frank escreveu, no seu célebre A miséria do povo, mãe das enfermidades (De populorum miseria: morborum genitrices), que pobreza e más condições de vida, trabalho e desnutrição eram as principais causas das doenças. Preconizou, mais do que reformas sanitárias, amplas reformas sociais e econômicas ${ }^{5}$. Chadwi$\mathrm{ck}^{6}$, na primeira metade do século XIX, referindo-se à situação de saúde dos ingleses, afirmava que ela era afetada pelo ambiente social e físico, e que a pobreza era muitas vezes consequência de doenças pelas quais os indivíduos não podiam ser responsabilizados, sendo um fator importante no aumento do número de pobres. Segundo Sigerist ${ }^{5}$, Chadwick não queria apenas aliviar os efeitos das condições de vida e saúde dos pobres ingleses, mas sim transformar suas causas.

Há muito tempo tem sido questionado o papel da medicina, da saúde pública e do setor saúde no enfrentamento do que seriam as causas mais amplas e gerais dos problemas de saúde, ou seja, as que fogem à atuação médica propriamente dita. Rudolf Virchow, na Alemanha, por exemplo, nos anos que precederam a revolução de 1848 , liderou um poderoso movimento de reforma médica defendendo que a medicina é uma ciência social. E a política não é mais do que a medicina em grande escala ${ }^{5}$.

Em um livro já clássico, McKeown \& Lowe $^{7}$ afirmam que a melhoria na nutrição e no saneamento e as modificações nas condutas da reprodução humana (sobretudo a diminuição no número de filhos por família) foram responsáveis pela redução da mortalidade na Inglaterra e no País de Gales, no século XIX e na primeira metade do século XX. As intervenções médicas eficazes, como as imunizações e a antibioticoterapia tiveram influência tardia e de importância relativa. No panorama mundial, estudos clássicos, como o 'Black Report' inglês de 1980, além de uma notável tradição de estudos canadenses, norte-americanos e europeus, são pródigos em mostrar as relações entre saúde e qualidade/condições de vida.

O debate sobre qualidade/condições de vida e saúde tem também razoável tradição tanto no Brasil quanto na América Latina. Paim ${ }^{8}$ publicou uma revisão sobre estudos da área de medicina e epidemiologia social que relacionavam condições de vida e saúde. O autor destaca os trabalhos pioneiros de Josué de Castro, Samuel Pessoa, Hugo Behm Rosas (Chile), e outros mais recentes, como os de Breilh e Gandra, no Equador, Laurell, no México e Monteiro, Possas, Arouca e do próprio autor, no Brasil. Estudos publicados, a partir do ano 2000, na Revista Ciência \& Saúde 
Coletiva também destacam a relação entre promoção da saúde e qualidade de vida ${ }^{9-25}$.

A Associação Brasileira de Saúde Coletiva (Abrasco) realizou em 2000 um seminário sobre condições de vida e situação de saúde em que vários autores latino-americanos discutiram o tema da saúde e qualidade de vida. O mesmo vem ocorrendo nos congressos brasileiros de saúde coletiva, epidemiologia e ciências sociais e saúde promovidos pela mesma entidade, em diferentes conjunturas. Duas revisões, publicadas simultaneamente ${ }^{26,27}$, exploram diversas dimensões do tema. Em 2019, dados da OPAS $^{3}$ indicavam os avanços na melhoria da saúde da população da Região das Américas, ao mesmo tempo em que destacam o trabalho que ainda há pela frente. Particularmente, as intervenções que podem e devem partir do setor saúde.

$\mathrm{Na}$ articulação entre saúde e condições e qualidade de vida, pode-se identificar, com flagrante inspiração dos pensadores e dos movimentos pioneiros da saúde pública e da medicina social, o desenvolvimento da promoção da saúde como campo conceitual e de práticas. É o que se discutirá a seguir.

\section{Promoção da saúde}

Promoção da saúde, como vem sendo entendida nos últimos 30-35 anos, representa uma estratégia promissora para enfrentar os problemas de saúde que afetam as populações humanas. Partindo de uma concepção ampla do processo saúde-doença e de seus determinantes, essa estratégia propõe a articulação de saberes técnicos e populares, e a mobilização de recursos institucionais e comunitários, públicos e privados a favor da qualidade de vida.

Decorridos pouco mais de trinta anos da divulgação da Carta de Ottawa ${ }^{28}$, um dos documentos fundadores do conceito contemporâneo de promoção da saúde, esse termo ficou associado a um conjunto de valores: qualidade de vida, solidariedade, equidade, democracia, cidadania, desenvolvimento, participação e parceria, entre outros. Refere-se também a uma combinação de estratégias: do Estado (políticas públicas saudáveis), da comunidade (reforço da ação comunitária), dos indivíduos (desenvolvimento de habilidades pessoais), do sistema de saúde (reorientação das estratégias) e de parcerias intersetoriais. Promoção da saúde trabalha com a ideia de responsabilidade múltipla pelos problemas e pelas soluções.

A promoção da saúde reage à acentuada medicalização da vida social e é uma resposta seto- rial articuladora de diversos recursos técnicos e posições ideológicas. Embora o termo tenha sido usado a princípio para caracterizar um nível de atenção da medicina preventiva ${ }^{29}$, seu significado foi mudando e passou a representar também um enfoque político e técnico em torno do processo saúde-doença-cuidado.

O conceito moderno de promoção da saúde se desenvolveu, de forma mais vigorosa nos últimos trinta anos, nos países desenvolvidos, particularmente no Canadá, Estados Unidos e países da Europa Ocidental. Nove Conferências Internacionais ${ }^{30}$ sobre o tema, realizadas nos últimos 34 anos - em Ottawa (WHO, 1986), Adelaide (WHO, 1988), Sundsvall (WHO, 1991), Jacarta (WHO, 1997), México (2000), Bangkok (2005), Nairóbi (2009), Helsinki (2013) e Xangai (2016) - aprofundaram suas bases conceituais e políticas. Na América Latina, em 1992, realizou-se a Conferência Internacional de Promoção da Saú$\mathrm{de}^{31}$, trazendo formalmente o tema para o contexto sub-regional.

Sigerist apud Rosen ${ }^{6}$ foi um dos primeiros autores a usar o termo, quando definiu as quatro tarefas essenciais da medicina: promoção da saúde, prevenção de doenças, recuperação dos enfermos e sua reabilitação. O autor afirmou que a saúde se promove proporcionando condições de vida decentes, boas condições de trabalho, educação, cultura física e formas de lazer e descanso, para o que pediu o esforço coordenado de políticos, setores sindicais e empresariais, educadores e médicos.

Leavell \& Clark $^{29}$ utilizaram o conceito de promoção da saúde ao desenvolverem o modelo da história natural da doença, propondo três níveis e cinco etapas nas quais se poderiam aplicar medidas preventivas. Prevenção primária, com medidas destinadas a desenvolver a saúde como proteção específica do ser humano contra agentes patológicos ou por meio de barreiras ambientais. A educação em saúde é elemento importante para esse objetivo, assim como: bom padrão de nutrição; desenvolvimento adequado da personalidade; educação dos pais; educação sexual e aconselhamento pré-nupcial; moradia adequada; recreação; condições favoráveis no lar e no trabalho; exames de saúde periódicos e aconselhamento e contato dos médicos com seus pacientes.

Promoção da saúde, nos moldes citados revelou-se insuficiente para o enfrentamento das doenças crônicas não transmissíveis. Com a segunda revolução epidemiológica ${ }^{32}$, as estratégias de cuidado passaram a associar-se a medidas preventivas sobre o ambiente físico e sobre os estilos 
de vida, e não exclusivamente à situação dos indivíduos e das famílias.

As diversas conceituações de promoção da saúde podem ser reunidas em dois grandes gru$\operatorname{pos}^{33}$. O primeiro diz respeito a atividades dirigidas à transformação dos comportamentos individuais, localizando-os no seio das famílias e nos ambientes comunitários. Nesse caso, os programas tendem a concentrar-se em componentes educativos relacionados a riscos comportamentais passíveis de mudanças e sob controle das pessoas, como o hábito de fumar, a dieta gordurosa, o sedentarismo e a direção perigosa ${ }^{29}$.

O segundo grupo enfatiza o papel protagonista dos determinantes gerais sobre as condições de saúde e se sustenta no entendimento de um amplo espectro de fatores como alimentação, habitação e saneamento; condições de trabalho; oportunidades de educação ao longo da vida; ambiente físico; apoio social para famílias e indivíduos; estilo de vida responsável; e cuidados de saúde. As estratégias são consideradas fruto de políticas e de condições favoráveis ao desenvolvimento da saúde por meio de escolhas saudáveis e reforço na capacidade de ação dos indivíduos e das comunidades. Ele surgiu formalmente no Canadá, em maio de 1974, com a divulgação do documento A New Perspective on the Health of Canadians, também conhecido como Informe Lalonde $^{34}$. Lalonde era então ministro da Saúde daquele país. A motivação central do documento parece ter sido política, técnica e econômica, pois visava a enfrentar os custos crescentes da assistência médica, ao mesmo tempo em que se apoiava no questionamento da abordagem exclusivamente médica para as doenças crônicas, com poucos resultados significativos.

Os fundamentos do Informe Lalonde encontram-se no conceito de campo da saúde, que reúne os chamados determinantes da saúde e contempla quatro componentes: biologia humana, ambiente, estilo de vida e organização da assistência à saúde. Nesse documento, o autor concluiu que quase todos os esforços da sociedade canadense destinados a melhorar a saúde, bem como a maior parte dos gastos diretos em matéria de saúde, concentravam-se na organização da assistência médica. No entanto, as causas principais das enfermidades e mortes tinham origem na biologia humana, no meio ambiente e nos estilos de vida.

Em 1978, a Organização Mundial da Saúde (OMS) convocou, em colaboração com o Fundo das Nações Unidas para a Infância (UNICEF), a I Conferência Internacional sobre Cuidados Pri- mários de Saúde, que se realizou em Alma-Ata ${ }^{35}$. A conferência colocou a meta de "saúde para todos no ano 2000" e recomendou oito pontos considerados essenciais para atingir tal objetivo: educação dirigida aos problemas de saúde prevalentes e métodos para sua prevenção e controle; promoção de suprimento de alimentos e nutrição adequada; abastecimento de água e saneamento básico; atenção materno infantil e planejamento familiar; imunização contra as principais doenças infecciosas; prevenção e controle de doenças endêmicas; tratamento apropriado de doenças comuns e acidentes; e distribuição de medicamentos básicos.

Talvez o que tenha ficado como marca dessa conferência tenha sido a proposta da Atenção Primária à Saúde (APS). Mas outros pontos menos divulgados devem ser ressaltados: reafirmação da saúde como direito; repúdio às desigualdades sociais; necessidade de nova ordem econômica mundial; responsabilidade dos governos pela saúde dos cidadãos; e direito de a população participar das decisões no campo da saúde. As conclusões e as recomendações de Alma Ata trouxeram importante reforço para os defensores das estratégias de promoção da saúde, o que culminou na realização da I Conferência Internacional sobre Promoção da Saúde, em Ottawa, Canadá, em 1986.

Como já dito, essa segunda concepção resgata, ainda que de modo distinto, as proposições de sanitaristas do século XIX, como Villermé, na França, Chadwick, na Inglaterra e Virchow e Neumann, na Alemanha, para quem as causas das epidemias eram tanto sociais e econômicas como físicas, e os remédios para as mesmas, prosperidade, educação e liberdade ${ }^{32}$.

Hartz $^{36}$ comenta que a promoção pode ser considerada também um eixo a ser privilegiado na formação, investigação e avaliação no campo da saúde pública visando à reorganização do sistema de cuidados e de articulação intersetorial. A importância da integração recursiva de práticas-formação-pesquisa, mediando-se pelo "conceito" de espaço sociossanitário nos estudos avaliativos tem servido para documentar colaborações de diversos setores e esferas de atividade, e para mostrar conflitos, diferenciais de recursos e poder e as desigualdades sociais. No entanto, a busca de solução das controvérsias que emergem leva a inovações, organiza espaços de reflexividade e facilita a integração dos diferentes atores.

Gutierrez $^{37}$ numa leitura apropriada à América Latina, além dos elementos já citados, ressalta o papel da comunidade e a responsabilidade in- 
delegável do Estado. Em resumo, o conceito de promoção da saúde vem sendo elaborado por diferentes atores técnicos e sociais, em diferentes conjunturas e formações sociais. Nos Quadros 1 e 2 encontra-se uma breve (e certamente incompleta) cronologia do desenvolvimento do campo da promoção da saúde no mundo e no Brasil, conforme Buss ${ }^{38}$.

\section{Conferências Internacionais sobre Promoção da Saúde}

Conferência de Otawa - Com a participação de cerca de 38 países, principalmente do mundo industrializado, a I Conferência Internacional sobre Promoção da Saúde teve como principal produto a chamada Carta de Ottawa, que se tornou um termo de referência para as ideias de promoção da saúde em todo o mundo. Por esse motivo a ela será dado um espaço maior do que à descrição das outras ${ }^{30}$.

A Carta de Ottawa define promoção da saúde como o processo de capacitação da comunidade para atuar na melhoria da sua qualidade de vida e saúde, incluindo participação no controle deste processo. O documento ressalta que saúde é o maior recurso para o desenvolvimento social, econômico e pessoal de um povo, assim como importante dimensão de sua qualidade de vida, transcendendo à ideia de formas sadias de vida. Afirma também que as condições e os requisitos para a saúde são: paz, educação, habitação, alimentação, renda, ecossistema estável, recursos sustentáveis, justiça social e equidade. Defesa da saúde, capacitação e mediação são, segundo a Carta, as três estratégias fundamentais da promoção da saúde.

O documento afirma que a finalidade da promoção da saúde é assegurar igualdade de oportunidades e meios para que todas as pessoas realizem seu potencial humano: ambientes favoráveis, acesso à informação, habilidades para viver melhor e possibilidade de fazer escolhas saudáveis. Os profissionais da área social e de saúde têm responsabilidade de contribuir que tal finalidade seja atingida.

A Carta de Ottawa propõe cinco campos de ação: (1) elaboração e implementação de políticas públicas saudáveis; (2) criação de ambientes favoráveis à saúde; (3) reforço da ação comunitária; (4) desenvolvimento de habilidades pessoais; (5) reorientação do sistema de saúde. A implementação de políticas públicas saudáveis implica na construção da saúde como prioridade de políticos e dirigentes de todos os setores; materia- liza-se em legislação, medidas fiscais, taxações e mudanças organizacionais; e realiza-se por meio de ações intersetoriais que levem a equidade em saúde, a distribuição equitativa de renda e as políticas sociais inclusivas.

A criação de ambientes favoráveis à saúde implica no reconhecimento das relações de interdependência entre os setores: proteção do meio ambiente; acompanhamento do impacto das mudanças ambientais; conquista de direitos ao trabalho, lazer, moradia, escola, dentre outros; acesso à informação e oportunidades de aprendizagem; educação para a saúde em casa, na escola, no trabalho e em outros espaços coletivos.

O desenvolvimento de habilidades e atitudes pessoais favoráveis à saúde em todas as etapas da vida envolve as instituições, particularmente as de educação e saúde, no empoderamento das pessoas por meio da aquisição de conhecimentos, acesso a bens e serviços e aumento do poder político dos indivíduos e da comunidade.

Para reorientação dos serviços de saúde, a Carta propõe a superação do modelo biomédico, centrado na doença e na assistência médica curativa. Os resultados esperados seriam transformações na organização e financiamento dos sistemas e serviços, dando ênfase à promoção da saúde e à formação dos profissionais já com outra mentalidade.

Conferência de Adelaide - A Conferência de Adelaide, realizada em 1988, focou-se nas políticas públicas saudáveis em todas as áreas, identificando a intersetorialidade e a responsabilização do setor público, não só pelas políticas sociais que faz ou deixa de fazer como também pelas políticas econômicas e seu impacto sobre a situação de saúde e no sistema de saúde. O documento final reafirmou a visão global e a responsabilidade internacionalista da promoção da saúde: estabeleceu que, devido ao fosso econômico-social e de saúde entre os países, os desenvolvidos têm obrigação de assegurar que suas políticas públicas resultem em impactos positivos na saúde das nações em desenvolvimento ${ }^{30}$.

Conferência de Sundsvall - Essa III Conferência, realizada na Suécia em 1991, foi a primeira a focar diretamente a interdependência entre saúde e ambiente. Ela ocorreu na efervescência prévia à primeira das grandes iniciativas das Nações Unidas previstas para preparar o mundo para o século XXI: a Conferência das Nações Unidas sobre Meio Ambiente e Desenvolvimento, a Rio92. Ampliava-se, aos poucos, a consciência dos indivíduos, movimentos sociais e governos sobre os riscos de um colapso do planeta diante das 
Quadro 1. Promoção da Saúde: uma breve cronologia.

1974 - Informe Lalonde: Uma Nova Perspectiva sobre a Saúde dos Canadenses/ A New Perspective on the Health of Canadians

1976 - Prevenção e Saúde: Interesse de Todos, DHSS (Grã-Bretanha)

1977 - Saúde para Todos no Ano 2000 - 30a Assembleia Mundial de Saúde

1978 - Conferência Internacional sobre Atenção Primária de Saúde - Declaração de Alma-Ata

1979 - População Saudável/Healthy People: The Surgeon General's Report on Health Promotion and Disease

Prevention, US-DHEW (EUA)

1980 - Relatório Black sobre as Desigualdades em Saúde/Black Report on Inequities in Health, DHSS (Grã-Bretanha)

1984 - Toronto Saudável 2000 - Campanha lançada no Canadá

1985 - Escritório Europeu da Organização Mundial da Saúde: 38 Metas para a Saúde na Região Europeia

1986 - Alcançando Saúde para Todos: Um Marco de Referência para a Promoção da Saúde/ Achieving Health for

All: A Framework for Health Promotion - Informe do Ministério da Saúde do Canadá.

Carta de Ottawa sobre Promoção da Saúde - I Conferência Internacional sobre Promoção da Saúde (Canadá)

1986- VIII Conferência Nacional de Saúde (Brasil)

1987 - Lançamento pela OMS do Projeto Cidades Saudáveis

1988 - Declaração de Adelaide sobre Políticas Públicas Saudáveis - II Conferência Internacional sobre Promoção da Saúde (Austrália).

De Alma-Ata ao ano 2000: Reflexões no Meio do Caminho - Reunião Internacional promovida pela OMS em Riga

(URSS)

Brasil - Constituição Brasileira, artigo 196: A saúde é direito de todos e dever do Estado, garantido mediante políticas sociais e econômicas que visem à redução do risco de doença e de outros agravos e ao acesso universal e igualitário às ações e serviços para sua promoção, proteção e recuperação.1989 - Uma Chamada para a Ação -

Documento da OMS sobre promoção da saúde em países em desenvolvimento

1990 - Cúpula Mundial das Nações Unidas sobre a Criança, NY.

1991 - Declaração de Sundsvall sobre Ambientes Favoráveis à Saúde - III Conferência Internacional sobre

Promoção da Saúde (Suécia)

1992 - Conferência das Nações Unidas sobre Meio Ambiente e Desenvolvimento (Rio-92)

Declaração de Santa Fé de Bogotá - Conferência Internacional sobre Promoção da Saúde na Região das Américas

(Colômbia)

1993 - Carta do Caribe para a Promoção da Saúde - I Conferência de Promoção da Saúde do Caribe (Trinidad e

Tobago)

Conferência das Nações Unidas sobre os Direitos Humanos (Viena)

1994 - Conferência das Nações Unidas sobre População e Desenvolvimento (Cairo)

1995 - Conferência das Nações Unidas sobre a Mulher (Pequim)

Conferência das Nações Unidas sobre o Desenvolvimento Social (Copenhague)

1996 - Conferência das Nações Unidas sobre Assentamentos Humanos (Habitat II) (Istambul)

1997 - Cúpula Mundial das Nações Unidas sobre Alimentação (Roma)

1997 - Declaração de Jacarta sobre Promoção da Saúde no Século XXI em diante - IV Conferência Internacional

sobre Promoção da Saúde (Indonésia) 2000 - V Conferência Internacional sobre Promoção da Saúde (Cidade do

México)

2000 - Conferência das Nações Unidas sobre os Objetivos de Desenvolvimento do Milênio

2005 - VI Conferência Internacional sobre Promoção da Saúde (Bangkok)

2006 - Política Nacional de Promoção da Saúde (Brasil)

2008 - Informe da Comissão Global sobre Determinantes Sociais da Saúde (OMS)

2009 - VII Conferência Mundial sobre Promoção da Saúde (Nairóbi)

2012 - Conferência das Nações Unidas sobre Meio Ambiente e Desenvolvimento (Rio +20) (Brasil)

2011 - Conferência Mundial sobre Determinantes Sociais da OMS (Rio de Janeiro)

2013 - VIII Conferência Mundial de Promoção da Saúde: Saúde em Todas as Políticas (Helsinque)

2014 - Política Nacional de Promoção da Saúde (Brasil), versão revisada e atualizada

2015 - Conferência das Nações Unidas sobre os Objetivos de Desenvolvimento Sustentável (ODS) e lançamento da Agenda 2030

2016 - IX Conferência Mundial de Promoção da Saúde (Shangai)

2018 - Conferência Global de Atenção Primária à Saúde (Astana)

2019 - Reunião de Alto Nível das Nações Unidas sobre Cobertura Universal em Saúde. (VERIFICAR)

Fonte: Atualizado pelos autores a partir do artigo original de Buss ${ }^{38}$ 
Quadro 2. Promoção da Saúde no Brasil: década a década - 1970-2010.

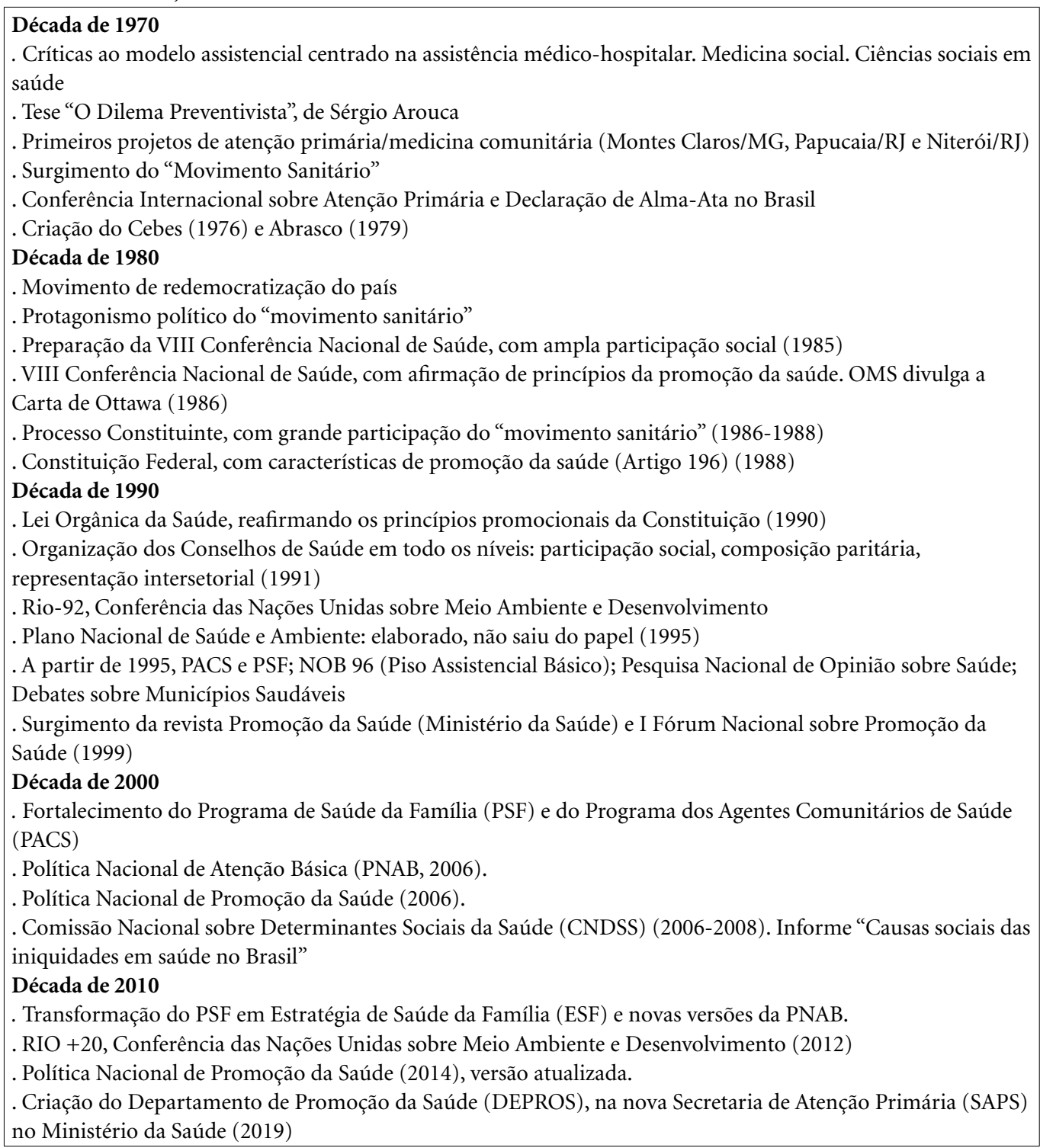

Fonte: Atualizado pelos autores a partir do artigo original de Buss ${ }^{38}$.

inúmeras e profundas agressões ambientais decorrentes do modo de produção e consumo das sociedades contemporâneas. $\mathrm{O}$ evento trouxe $\mathrm{o}$ tema do ambiente não restrito à dimensão física ou natural, mas também social, econômica, política e cultural. Referiu-se claramente aos espaços onde as pessoas vivem: a comunidade, suas casas, seu trabalho, seus espaços de lazer e as estruturas econômicas e políticas que determinam o acesso aos recursos para viver e tomar decisões. Sublinhou quatro aspectos: (1) a dimensão social, na qual se incluem normas, costumes e proces- sos sociais que afetam a saúde e alertou para as quebras nas relações tradicionais pelo crescente isolamento social e perda de valores; (e) a dimensão política que requer dos governos a garantia de participação democrática nos processos de decisão e descentralização de recursos e de responsabilidades, (3) a dimensão econômica, que sublinha a necessidade de escalonar recursos para setores sociais, de saúde e desenvolvimento sustentável; (4) a utilização da capacidade e conhecimento das mulheres, inclusive nos setores político e econômico ${ }^{30}$. O documento fez menção 
a experiências locais oriundas de todo o mundo, formando cenários para ação na denominada pirâmide dos ambientes favoráveis de Sundsvall: educação, alimentação e nutrição, moradia e vizinhanças, apoio e atenção social, trabalho e transporte. Tais experiências foram reunidas e revisadas num informe da $\mathrm{OMS}^{39}$.

Conferência de Jacarta - Esta foi a primeira Conferência a se realizar num país em desenvolvimento. Desde seu subtítulo "Novos atores para uma nova era", nela se reforçou a ação comunitária como uma dimensão fundamental da promoção da saúde. A conferência reforçou as cinco estratégias descritas na Carta de Ottawa, mostrando que as ações são mais eficazes quando centradas simultaneamente em todas. Foram definidas cinco prioridades para os anos subsequentes: (1) promover a responsabilidade social com a saúde, por meio de políticas públicas saudáveis e comprometimento do setor privado; (2) investimentos no setor saúde de forma articulada com as áreas de educação, habitação e sociais; (3) consolidar e expandir parcerias em todos os níveis de governo e da sociedade; (4) aumentar a capacidade da comunidade e fortalecer os indivíduos por meio da educação, capacitação de lideranças e acesso a recursos; (5) definir cenários preferenciais para atuação (escolas, ambientes de trabalho e outros) ${ }^{30}$.

Declaração de Bogotá - Assim é conhecido o documento que lançou a proposta de promoção da saúde na América Latina. Nele se afirma que a Região deve criar condições que garantam o bem-estar geral como propósito fundamental do desenvolvimento. Parte da análise de que, assolada pelas desigualdades agravadas pela prolongada crise econômica e pelas políticas de ajuste macroeconômico a América Latina enfrenta a deterioração das condições de vida da maioria da população, aumento dos riscos para a saúde e redução de recursos para enfrentá-los. O desafio da promoção da saúde inclui transformar as relações excludentes, conciliando interesses econômicos, propósitos sociais, solidariedade e equidade social ${ }^{31}$. O documento estabelece cinco princípios ou premissas:

(1) Superação das desigualdades econômicas, ambientais, sociais, políticas e culturais; e as relativas à cobertura, acesso e qualidade dos serviços de saúde. (2) Alternativas orientadas a superar, simultaneamente, as enfermidades causadas pelo atraso e a pobreza e as derivadas da urbanização e da industrialização. (3) Reafirmação da democracia nas relações políticas e sociais. (4) Conquista da equidade, pela identificação dos fatores que favorecem a iniquidade e propostas de ação para aliviar seus efeitos; (5) Desenvolvimento integral dos seres humanos e das sociedades. Esse documento apontou três estratégias para a promoção da saúde na Região: (1) impulsionar a cultura da saúde, modificando valores, crenças, atitudes e relações; (2) dar relevo às estratégias de promoção da saúde: (3) convocar, animar e mobilizar um grande compromisso social para assumir a política de saúde como prioridade. E ainda definiu onze compromissos indispensáveis para alcançar os objetivos propostos:

(1) Impulsionar o conceito de saúde condicionado por fatores políticos, econômicos, sociais, culturais, ambientais, comportamentais e biológicos, tendo a promoção da saúde como estratégia; (2) Convocar as forças sociais para aplicar a estratégia de promoção da saúde; (3) Incentivar políticas públicas de equidade e de ambientes e opções saudáveis; (4) Estabelecer mecanismos de concertação entre os setores sociais e institucionais; (5) Reduzir gastos improdutivos e a profusão de burocracias centralizadoras, fontes de ineficiência e desperdício; (6) Fortalecer a capacidade da população para participar nas decisões que afetam sua vida e para optar por estilos de vida saudáveis; (7) Eliminar os efeitos das desigualdades sobre a mulher; (8) Estimular o diálogo dos diversos saberes. (9) Fortalecer a capacidade de convocação do setor para mobilizar a produção social de saúde e mostrar as responsabilidades dos diferentes atores na sua construção; (10) Reconhecer como trabalhadores e agentes de saúde as pessoas comprometidas com os processos de promoção da saúde; (11) Estimular investigações em promoção da saúde, gerar ciência e tecnologia apropriada e disseminar conhecimentos.

Conferência do México - A V Conferência Internacional sobre Promoção da Saúde foi realizada na Cidade do México, no ano 2000 e teve como objetivos: (1) avaliar o impacto da promoção da saúde na saúde e qualidade de vida, especialmente, para as pessoas que vivem em circunstâncias adversas; (2) elevar a saúde a um lugar de destaque no programa de desenvolvimento das organizações internacionais, nacionais e locais; e (3) incentivar a formação de alianças para a saúde entre os diferentes setores e em todos os níveis da sociedade. Na Declaração intitulada "Das Ideias às Ações", destacam-se: colocar a promoção da saúde como prioridade nas políticas e programas locais, regionais, nacionais e internacionais; assegurar a participação ativa de todos os setores e da sociedade civil no desenvolvimento das ações de 
promoção; apoiar a preparação de planos de ação nacionais para promoção da saúde; e, estabelecer e fortalecer redes nacionais e internacionais que promovam a saúde ${ }^{30}$.

Conferência de Bangkok - Em 2005, essa VI Conferência acentuou os processos de globalização pelos quais o mundo passa e a promoção da saúde. Ao mesmo tempo em que se reconheceram as oportunidades de cooperação advindas das tecnologias de informação e comunicação e da ampliação de mecanismos eficientes de governança global e troca de experiências entre os países, foram assinalados problemas, como o aumento crescente das desigualdades dentro dos países e entre eles, novos padrões de consumo não saudáveis, mudanças ambientais e um processo crescente e desordenado de urbanização. $\mathrm{Na}$ Carta final, foi reiterada a necessidade da participação ativa da sociedade civil para o alcance da "Saúde para Todos" e estabelecidos quatro compromissos: (1) foco da agenda de desenvolvimento global; (2) responsabilidade central dos governos; (3) importância das comunidades e da sociedade civil; (4) investimentos nas boas práticas corporativas. Ao final do documento, os participantes fizeram um chamado à ação e solicitaram às Organizações das Nações Unidas que avaliassem os benefícios de estabelecer um Tratado Global para a Saúde ${ }^{30}$.

Conferência de Nairobi - A VII Conferência em 2009 foi a primeira realizada no continente africano. A Declaração do evento, denominada Call to Action reforçou a importância da promoção da saúde, de fortalecer lideranças, de investir na força de trabalho em saúde, de capacitar comunidades e indivíduos e de aprimorar os processos participativos $^{30}$.

Conferência de Helsinque - Essa VIII Conferência lançou o desafio de construir estratégias com foco em "Saúde em Todas as Políticas". Ela aprofundou as implicações das decisões de todas as áreas sobre a saúde e a busca de sinergias entre elas, a favor da equidade. A declaração do evento chamou a atenção das autoridades políticas sobre as consequências de suas decisões para o bem estar e a saúde das populações. Foram propostas as seguintes ações: (1) adotar o enfoque da Saúde em Todas as Políticas; (2) assegurar estruturas e processos sustentáveis que efetivem o referido enfoque; (3) fortalecer a capacidade dos Ministérios da Saúde para envolver outros setores do governo, por meio de liderança, parceria, advocacia e mediação, para alcançar resultados de saúde; (4) desenvolver recursos humanos, capacidade institucional e habilidades técnicas que facili- tem o objetivo de Saúde em Todas as Políticas; (5) adotar auditoria transparente e mecanismos de responsabilização que construam a confiança entre governos e cidadãos; (6) estabelecer salvaguardas contra os conflitos de interesse que prejudiquem a saúde nos investimentos comerciais; (7) fomentar o engajamento público e da sociedade civil no desenvolvimento, implementação e monitoramento da Saúde em Todas as Políticas ${ }^{30}$.

Conferência em Shangai - Esta IX Conferência foi realizada em 2016. Seu foco foi promover a saúde mediante a adoção de medidas condizentes e com o alcance dos Objetivos de Desenvolvimento Sustentável (ODS). Da Declaração do evento constam quatro grandes temas e uma série de compromissos: (1) adoção de decisões políticas em favor dos direitos das mulheres, das populações deslocadas e do crescente número de pessoas afetadas por crises humanitárias e ambientais. (2) Utilização de estratégias de governança para promover o bem-estar. (3) Reconhecimento das cidades e comunidades como ambientes essenciais à saúde. (4) Reconhecimento do saber em saúde como elemento fundamental para a promoção da equidade em saúde. A Declaração termina com um "chamado à ação" para que os compromissos assumidos acelerem a implementação dos ODS por meio do compromisso político e do investimento financeiro na promoção da saúde ${ }^{30}$.

\section{Políticas públicas saudáveis, intersetorialidade e municípios saudáveis}

No debate sobre promoção da saúde, especial destaque deve ser dado às políticas públicas saudáveis, à governabilidade, à gestão social integrada, à intersetorialidade, às estratégias dos municípios saudáveis e ao desenvolvimento local. Como já dito, não é novo o reconhecimento da contribuição das políticas públicas para a saúde das populações. Remonta aos primórdios do Estado moderno, por volta do século XVII, embora $\mathrm{o}$ advento da era microbiológica, em meados do século XIX, tenha restringido o escopo da ação sanitária, despojando-a de seu caráter de intervenção social e enfatizando seu caráter técnico e setorial.

Entretanto, de forma curiosa e até paradoxal, a relação entre políticas públicas e saúde volta a ganhar relevo nos últimos anos, não tanto por seus benefícios, mas pelos malefícios gerados. São notórios, e por vezes dramáticos, os efeitos das políticas que impulsionaram a economia urbano-industrial ao longo do século XX: desi- 
gualdades sociais, danos ambientais irreparáveis em alguns casos, ambientes sociais mórbidos de sociopatias e psicopatias, por exemplo.

A ideia contemporânea de políticas públicas saudáveis envolve um duplo compromisso: o de situar a saúde no topo da agenda pública, promovendo-a de setor da administração a critério de governo, e o compromisso técnico de enfatizar, como foco de intervenção, os fatores determinantes do processo saúde-doença-cuidado. Sua perspectiva ultrapassa em abrangência as ações ambientais da saúde pública tradicional e, mesmo, as políticas urbanas de expansão de serviços e bens de consumo coletivo. Implica numa (re) formulação inovadora, tanto do conceito de saúde, quanto do de Estado (e, portanto, de política pública) e de seu papel perante a sociedade.

A nova concepção de Estado, subjacente à proposta das políticas públicas saudáveis, é aquela que (re)estabelece a centralidade de seu caráter público e de sua responsabilidade social, isto é, seu compromisso com o interesse e o bem comum. Numa perspectiva de reforma do Estado, isso implica um esforço (desenho institucional) de superar déficits de eficiência/efetividade (capacidade de fazer o que deve ser feito) e de representatividade/sensibilidade (capacidade de definir o que precisa ser feito, segundo o interesse e as necessidades da sociedade).

Um ponto importante no marco da promoção da saúde é a superação da ideia de políticas públicas como iniciativas exclusivas ou monopolistas do aparelho estatal. Em fóruns participativos, expressivos da diversidade de interesses e necessidades sociais, as políticas públicas tendem a ser comprometidas com a saúde na linha de "saúde em todas as políticas".

Outro aspecto fundamental é o empoderamento da população organizada, através da difusão ampla das evidências sobre as relações entre saúde e seus pré-requisitos, assim como da construção de mecanismos de atuação eficientes. Numa nova distribuição de deveres e direitos entre o Estado e a sociedade, entre indivíduos e coletivos, entre público e privado, a questão da participação é pré-requisito institucional e político para a definição da "saúde que queremos".

Um cuidado importante é evitar que a defesa de políticas públicas saudáveis implique na subordinação de outros setores governamentais, gerando resistências e suscitando isolamentos. Tendo na interdisciplinaridade o seu fundamento cognitivo e na intersetorialidade a sua ferramenta operacional, as políticas saudáveis, para não se limitarem a uma normatividade burocrática socialmente natimorta, devem suscitar ou partir de pactos horizontais com parceiros de outros setores governamentais e de outras comunidades epistêmicas, como urbanistas, educadores e ambientalistas. A intersetorialidade tão desejada pode ser definida como o processo no qual objetivos, estratégias, atividades e recursos de cada setor são considerados segundo suas repercussões e efeitos nos objetivos, estratégias, atividades e recursos dos demais setores ${ }^{31}$.

Forjar um Estado que opere na lógica da ação pública intersetorial supõe desenvolver uma nova institucionalidade social ${ }^{40}$, entendida como o conjunto de organismos estatais encarregados do desenho, coordenação, execução e financiamento das políticas sociais, inclusive a de saúde. Essa nova institucionalidade depende da configuração de uma autoridade social, ou o conjunto dos responsáveis pelas políticas sociais, que coordene as políticas intersetoriais e outros arranjos voltados ao desenvolvimento. Tal autoridade social deveria ter posição de mesmo nível na estrutura de poder que as autoridades econômicas, com funções de planejamento e execução claramente definidas, além de recursos financeiros garantidos na repartição orçamentária.

Diversos países no Continente têm procurado desenhos institucionais que articulem instâncias governamentais intra e intersetorialmente com a sociedade civil. No caso de países de estrutura federativa, como o Brasil, torna-se necessária também a coordenação entre os diversos níveis administrativos e os subsetores sociais. Um enfoque deste tipo requer necessariamente a criação de redes interinstitucionais e uma nova cultura organizacional que requer melhorar a qualidade dos recursos humanos envolvidos e gerar novas formas de relações e comunicação entre os distintos âmbitos do aparelho estatal ${ }^{40}$.

Nas últimas três décadas, no Brasil, uma série de experiências de gestão pública e mobilização social suscitou a implementação organizada de uma perspectiva de ação intersetorial, sob o rótulo do desenvolvimento local integrado e sustentável $^{41}$. Recentemente, impulsionadas pela Agenda 2030 e a definição dos Objetivos de Desenvolvimento Sustentável, iniciativas têm sido criadas com vistas ao alcance das metas propostas. A saúde pública latino-americana realmente tem condições de contribuir efetivamente para a construção teórica e prática de tais propostas, principalmente por meio da estratégia dos "Municípios Saudáveis", um modelo que contém os requisitos da formulação e implementação de políticas em prol da saúde por meio de ações intersetoriais. 
O movimento das cidades saudáveis surgiu na Europa, no mesmo ano (1986) em que se realizou a já mencionada Conferência de Ottawa. Segundo Ashton ${ }^{42}$, o projeto visa ao desenvolvimento de planos de ação locais para a promoção da saúde, baseados nos princípios de saúde para todos da OMS. A iniciativa hoje envolve muitas localidades, em várias redes nos cinco continentes. A constituição do programa num município inclui, em geral, quatro fases: início com determinação de prioridades; preparação de um plano de ação; unificação dos comitês organizadores e a execução de atividades e criação de sistemas de informação para o monitoramento e avaliação das iniciativas.

Referendado na Declaração de Santa Fé de Bogotáa $^{31}$ pela maioria dos países latino-americanos, o movimento dos municípios saudáveis chegou à América Latina no início da década de 1990. O movimento propõe uma reestruturação do sistema de saúde e de sua articulação com outros sistemas, na conformação de políticas e programas integrados para o desenvolvimento humano e o bem-estar. Segundo a OPAS ${ }^{43}$, esse enfoque se centra na ação e na participação, assim como na educação sanitária e na comunicação para a saúde, visando a ampliar a capacidade da comunidade de melhorar suas condições físicas e psicossociais nos espaços onde as pessoas vivem, estudam, trabalham e se divertem. Esse movimento avançou rapidamente na região das Américas na década de 1990, alcançando mais de 500 municípios em praticamente todos os países do Continente. Já foram realizados dois "Encontros Latino-Americanos de Secretarias Municipais de Saúde", um em Cuba (1994) e outro no Brasil (1996) para consolidar a iniciativa e trocar experiências.

A configuração dos municípios saudáveis varia em cada local, compreendendo desde programas setoriais e dirigidos à promoção de comportamentos individuais saudáveis até propostas bastante abrangentes que alcançam diversas dimensões e setores. Muitos países estabeleceram redes nacionais para o intercâmbio de experiências e para a busca de vantagens e incentivos nas negociações com os demais níveis do poder público. Um enorme desafio que ainda permanece é a identificação das melhores formas de institucionalidade social para uma gestão municipal integrada e participativa.

Por fim, um alerta: inovações tecnológicas ferramentas, serviços e plataformas digitais - têm grande potencial para contribuir com a promoção da saúde e prevenção de doenças. Soluções, como aplicativos, fóruns on-line, blogs, redes sociais, dentre outras novidades, podem ampliar as possibilidades de as pessoas se comunicarem a favor de uma vida saudável. Porém, assim como os meios digitais podem ampliar os benefícios, Relatório da União Europeia, publicado em $2019^{44}$, alerta que a falta de acesso, com segurança, aos recursos digitais e o desconhecimento para utilizar as ferramentas disponíveis pode exacerbar as iniquidades em saúde.

\section{Conclusões}

Os profissionais de saúde, os movimentos sociais e as organizações populares, políticos e autoridades públicas têm responsabilidades sobre as repercussões positivas ou negativas que as políticas públicas têm sobre a situação de saúde e as condições de vida. A estratégia dos municípios saudáveis é uma das iniciativas que pode propiciar, através de uma nova institucionalidade social, a promoção da saúde por intermédio da ação intersetorial.

Aqui, como em tudo no campo da saúde, não há receitas prontas. A mediação entre a população e o poder público, assim como a capacitação para o exercício da cidadania e do controle social são contribuições inestimáveis para a promoção da saúde que não podem ser descuidadas ou perdidas.

A mudança da legislação e a introdução de inovações nos Programas de Agentes Comunitários de Saúde e de Saúde da Família e a ampliação do piso assistencial básico podem ocasionar, no caso brasileiro, um extraordinário impulso à qualidade de vida e às condições de saúde, sob a ótica da promoção da saúde.

Compromissos globais, como a Agenda 2030, associadas com propostas de desenvolvimento local, podem contribuir enormemente para o estabelecimento de alianças pró-saúde e para inovações na gestão pública, em torno de processos como a intersetorialidade para políticas públicas saudáveis ${ }^{45}$.

A defesa da saúde e da promoção da saúde junto a políticos e aos movimentos da sociedade civil constituem uma forma de ativismo que compete a quem atua no setor e, aqui no Brasil, acredita no SUS. 


\section{Colaboradores}

PM Buss, ZMA Hartz, LF Pinto e CMF Rocha participaram igualmente de todas as etapas de atualização do artigo científico originalmente publicado em 2000 pelo primeiro autor. PM Buss revisou todo o texto.

\section{Referências}

1. World Health Organization (WHO). World Health Statistics 2019: monitoring health for the sustainable development goals. Genève: WHO; 2019.

2. Organização Pan-Americana da Saúde (OPAS). Sociedades justas: Equidade em saúde e vida com dignidade. Relatório da Comissão sobre Equidade e Desigualdades em Saúde nas Américas. Washington: OPAS; 2019.

3. Organização Pan-Americana da Saúde (OPAS). Indicadores básicos 2019: Tendencias de la salud en las Américas. Washington: OPAS; 2019.

4. Buss PM. Promoção da saúde e qualidade de vida. Cien Saude Colet 2000; 5(1):163-177.

5. Sigerist H. The University at the Crossroad. New York: Henry Schumann Publisher; 1956.

6. Rosen G. Da Polícia Médica à Medicina Social. Rio de Janeiro: Ed.Graal; 1979.

7. McKeown T, Lowe CR. Introducción a la Medicina Social. 4a ed. México: Siglo XXI; 1989.

8. Paim JS. Abordagens teórico-conceituais em estudos de condições de vida e saúde: notas para reflexão e Ação. In: Barata RB, organizador. Condições de Vida e Situação de Saúde. Saúde e Movimento. Rio de Janeiro: Abrasco; 1997. p. 7-30.

9. Andrade ER, Sousa ER, Minayo MCS. Intervenção visando a autoestima e qualidade de vida dos policiais civis do Rio de Janeiro. Cien Saude Colet 2009; 14(1):275-285.

10. Bittencourt ZZLC, Hoehne EL. Qualidade de vida de familiares de pessoas surdas atendidas em um centro de reabilitação. Cien Saude Colet 2009; 14(4):12351239.

11. Buss PM, Carvalho AI. Desenvolvimento da promoção da saúde no Brasil nos últimos 20 anos (19882008). Cien Saude Colet 2009; 14(6):2305-2316.

12. Maciel ELN, Oliveira CB, Frechiani JM, Sales CMM, Brotto LDA, Araújo MD. Projeto Aprendendo Saúde na Escola: a experiência de repercussões positivas na qualidade de vida e determinantes da saúde de membros de uma comunidade escolar em Vitória, Espírito Santo. Cien Saude Colet 2010; 15(2):389-396.

13. Campolina AG, Dini PS, Ciconelli RM. Impacto da doença crônica na qualidade de vida de idosos da comunidade em São Paulo. Cien Saude Colet 2011; 16(6):2919-2925.

14. Barbosa TS, Gavião MBD. Qualidade de vida e saúde bucal em crianças - parte II: versão brasileira do Child Perceptions Questionnaire. Cien Saude Colet 2011; 16(7):3267-3276.

15. Soares AHR, Martins AJ, Lopes MCB, Britto JAA, Oliveira CQ, Moreira MCN. Qualidade de vida de crianças e adolescentes: uma revisão bibliográfica. Cien Saude Colet 2011; 16(7):3197-3206.

16. Miotto MHMB, Barcellos LA, Velten DB. Avaliação do impacto na qualidade de vida causado por problemas bucais na população adulta e idosa em município da Região Sudeste. Cien Saude Colet 2012; 17(2):397-405.

17. Silveira MF, Almeida JC, Freire RS, Ferreira RC, Martins AEBL, Marcopito LF. Qualidade de vida entre adolescentes: estudo seccional empregando o SF-12. Cien Saude Colet 2013; 18(7):2007-2015. 
18. Silva CS, Bodstein RCA. Referencial teórico sobre práticas intersetoriais em Promoção da Saúde na Escola. Cien Saude Colet 2016; 21(6):1777-1788.

19. Miranda LCS, Soares SM, Silva PAB. Qualidade de vida e fatores associados em idosos de um Centro de Referência à Pessoa Idosa. Cien Saude Colet 2016; 21(11):3533-3544.

20. Fernandes AP, Andrade ACS, Costa DAS, Dias MAS, Malta DC, Caiaffa WT. Programa Academias da Saúde e a promoção da atividade física na cidade: a experiência de Belo Horizonte, MG. Cien Saude Colet 2017; 22 (12):3903-3914.

21. Agatha BT, Reichenheim ME, Moraes CL. Qualidade de vida relacionada à saúde de adolescentes escolares. Cien Saude Colet Rio de Janeiro, 2018, 23(2):659-668.

22. Barbosa ML, Menezes TN, Santos SR, Olinda RA, Costa GMC. Qualidade de vida no trabalho dos profissionais de saúde no sistema prisional. Cien Saude Colet 2018; 23(4):1293-1302.

23. Klein SK, Fofonka A, Hirdes A, Jacob MHVM. Qualidade de vida e níveis de atividade física de moradores de residências terapêuticas do sul do Brasil. Cien Saude Colet 2018; 23(5):1521-1530.

24. Dias ACB, Chaveiro N, Porto CC. Qualidade de vida no trabalho de fisioterapeutas docentes no município de Goiânia, Goiás, Brasil. Cien Saude Colet 2018; 23(9):3021-3030.

25. Sanchez HM, Sanchez EGM, Barbosa MA, Guimarães EC, Porto CC. Impacto da saúde na qualidade de vida e trabalho de docentes universitários de diferentes áreas de conhecimento. Cien Saude Colet 2019; 24(11):4111-4123.

26. Minayo MCS, organizadora. Os Muitos Brasis: Saúde e População na Década de 1980. São Paulo, Rio de Janeiro: Hucitec, Abrasco; 1995.

27. Monteiro CA, organizador. Velhos e Novos Males da Saúde no Brasil: A Evolução do País e de suas Doenças. São Paulo: Hucitec-Nupens/USP; 1995.

28. Brasil. Ministério da Saúde (MS). Declaração de Santa Fé de Bogotá. Promoção da Saúde: Cartas de Ottawa, Adelaide, Sundsvall e Santa Fé de Bogotá. Brasília: MS, Fiocruz; 1996. p. 41-47.

29. Leavell H, Clark EG. Medicina Preventiva. São Paulo: McGraw-Hill Inc.; 1976.

30. World Health Organization (WHO). WHO Global Health Promotion Conferences. [acessado 2020 maio 13]. Disponível em: https://www.who.int/healthpromotion/conferences/en/

31. Organização Pan-Americana da Saúde (OPAS). Caribbean Charter for Health Promotion. Washington: OPAS; 1993.

32. Terris M. Conceptos de la promoción de la salud: Dualidades de la teoría de la salud publica. In: Organização Pan-Americana da Saúde (OPAS). Promoción de la Salud: Una Antología. Washington: OPAS; 1996. p. 37-44.

33. Sutherland RW, Fulton MJ. Health promotion. In: Sutherland RW, Fulton MJ. Health Care in Canada. Ottawa: CPHA; 1992. p. 161-181.
34. Lalonde M. A new perspective on the health of $\mathrm{Ca}$ nadians. In: Organização Pan-Americana da Saúde (OPAS). Promoción de la Salud: Una Antología. Washington: OPAS; 1996. (Publ. Cient. 557) p. 3-5.

35. World Health Organization (WHO). Primary Health Care-Report of the International Conference on PHC, Alma-Ata. Genève: WHO; 1978.

36. Hartz ZMA. Cuidados primários, avaliação e ações intersetoriais em promoção da saúde. Cien Saude Colet 2017; 22(3):687-688.

37. Gutierrez M. Perfil descriptivo-situacional del sector de la promoción y educación en salud: Colombia. In: Arroyo HV, Cerqueira MT, editores. La Promoción de la Salud y la Educación para la Salud en America Latina: un Analisis Sectorial. San Juan: Editorial de la Universidad de Puerto Rico: 1996. p. 107-128.

38. Buss PM. Promoção da Saúde e Saúde Pública. Rio de Janeiro: ENSP; 1988. (mimeo).

39. Hanglund BJA, Pettersson B, Finer D, Tillgren P. Creating supportive environments for health. Public Health in Action 3. Genève: WHO; 1996.

40. Comissão Econômica para a América Latina e o Caribe (CEPAL) Panorama Social da América Latina 1997: Agenda Social. Santiago do Chile: CEPAL; 1998.

41. Comunidade Solidária Desenvolvimento local integrado e sustentável (DLIS). Documento básico e catálogo de experiências. Cadernos do Comunidade Solidária, 6. Brasília: IPEA; 1998.

42. Ashton J. Ciudades Sanas. Barcelona: Masson; 1993.

43. Organização Pan-Americana da Saúde (OPAS). Promoción de la Salud: Una Antología. Washington: OPAS; 1996. (Publ. Cient. 557)

44. European Union. State of Health in the EU: Companion Report 2019. Luxembourg: Publications Office of the European Union; 2019.

45. Buss PM. Saúde na Agenda do Desenvolvimento Sustentável 2030 e seus ODS: Análise e perspectivas da implementação na América Latina e Caribe (ALC) (20122019) [tese]. São Paulo: Universidade de São Paulo; 2020.

Artigo apresentado em 21/05/2020

Aprovado em 21/05/2020

Versão final apresentada em 23/05/2020 
